\title{
The Compton Spectrometer and Imager (COSI)
}

\author{
J.-L. Chiu*, S. E. Boggs, C. A. Kierans, A. Lowell, C. Sleator, J. A. Tomsick, A. \\ Zoglauer \\ Space Sciences Laboratory, University of California, Berkeley, CA 94720, USA \\ E-mail: alanchiuessl.berkeley.edu, clchiu214@gmail.com
}

M. Amman

Lawrence Berkeley National Laboratory, 1 Cyclotron Road, Berkeley, CA, USA

\author{
H.-K. Chang, C.-Y. Chu, C.-H. Tseng, C.-Y. Yang
}

Institute of Astronomy, National Tsing Hua University, Hsinchu 30013, Taiwan

C.-H. Lin

Institute of Physics, Academia Sinica, Taipei 11529, Taiwan

\section{P. Jean, P. von Ballmoos}

IRAP Toulouse, 9 avenue du Colonel Roche, Toulouse, France

\begin{abstract}
The Compton Spectrometer and Imager (COSI) is a balloon-borne, soft gamma-ray (0.2-5 MeV) telescope designed to study astrophysical sources of nuclear-line emission and gamma-ray polarization. The heart of COSI is a compact array of cross-strip germanium detectors (GeDs), providing excellent spectral resolution $(0.3 \%$ at $662 \mathrm{keV})$ and the capability of tracking the photon scattering history with full 3D position resolution of less than $2 \mathrm{~mm}^{3}$ for each interaction. With good efficiency, wide-field imaging, and polarization sensitivity, COSI is a powerful observatory in the medium gamma-ray regime. The most recent balloon flight of COSI was launched from Wanaka, New Zealand, in May 2016 on a Super Pressure Balloon floating for 46 days. During this flight, COSI discovered GRB 160530A and detected several sources, including the Crab, Cen A, Cyg X-1, and the 511-keV emission from the galactic center. Here we will present the characteristics and capabilities of this novel instrument and preliminary results from the 2016 flight.
\end{abstract}

35th International Cosmic Ray Conference - ICRC2017

10-20 July, 2017

Bexco, Busan, Korea

${ }^{*}$ Speaker. 


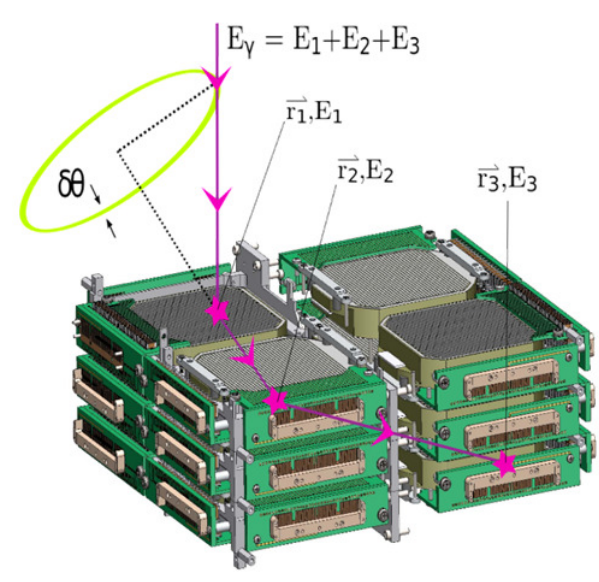

(a) Principle of Compton imaging

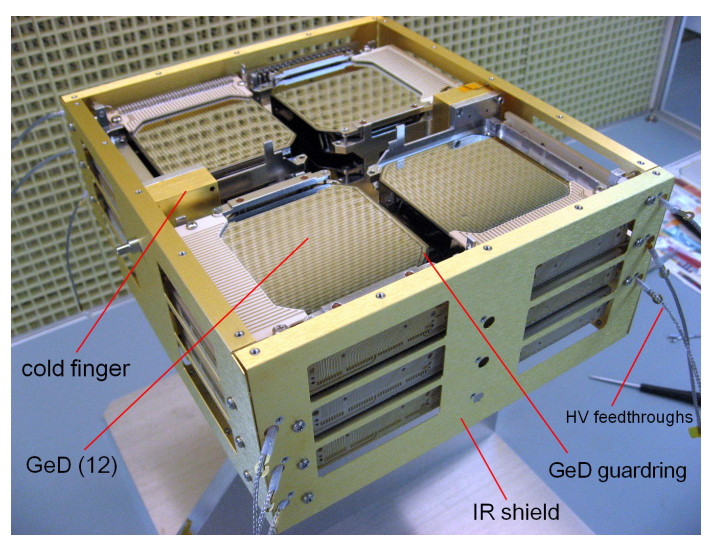

(b) COSI germanium detector aray

Figure 1: The principle and heart of COSI: (a) Principle of Compton imaging showing an example of a photon experiencing two Compton scatters followed by a full photo-absorption in the GeD array; the origin of the photon can be restricted to an annulus on the sky; (b) the twelve detector array before integration in the cryostat.

\section{Introduction}

The Compton Spectrometer and Imager (COSI) is a balloon-borne soft gamma-ray telescope (0.2-5 MeV) designed to study astrophysical sources of nuclear-line emission and gamma-ray polarization [1]. A compact Compton telescope design is employed by COSI to utilize novel technologies that are built upon Compton imaging techniques (Fig. 1(a)) successfully pioneered by COMPTEL on CGRO [2]. The heart of COSI is a compact array of 12 cross-strip germanium detectors (GeDs) (Fig. 1(b)) enclosed in a cryostat. The lower side of the cryostat is surrounded by anticoincidence cesium iodide (CsI) shields (Fig. 2(a)), resulting in a wide field-of-view (FoV) covering $\sim 1 / 4$ of the full sky at any given moment. COSI is a powerful observatory in soft gammaray regime with advanced capabilities beyond COMPTEL. The characteristics of COSI as well as a comparison between COSI and COMPTEL can be found in [3].

COSI is built upon considerable heritage from the Nuclear Compton Telescope (NCT), which successfully achieved two conventional balloon flights $[4,5]$ and reported the first astrophysical source detected with a compact Compton telescope [6]. After an upgrade optimized for the Super Pressure Balloon (SPB) platform, COSI became the first science payload to fly with NASA's new SPB platform from Antarctica in 2014 [3]; however, a leak in the balloon terminated this flight after only 43 hours. On May 17, 2016, COSI was launched from Wanaka, New Zealand. Staying afloat for 46 days, COSI achieved a new duration record for mid-latitude flights. In this paper, we will provide an overview of COSI's primary science goals, the instrument and the 2016 balloon flight, followed by our current progress of scientific analysis.

\section{Primary Science Goals}

Current telescope sensitivities in the soft/medium gamma-ray band is relatively poor compared 


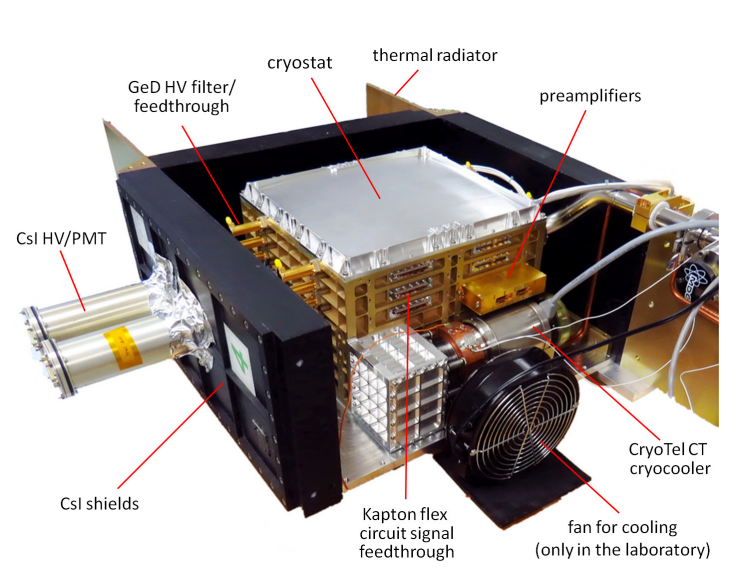

(a) COSI cryostat with shields

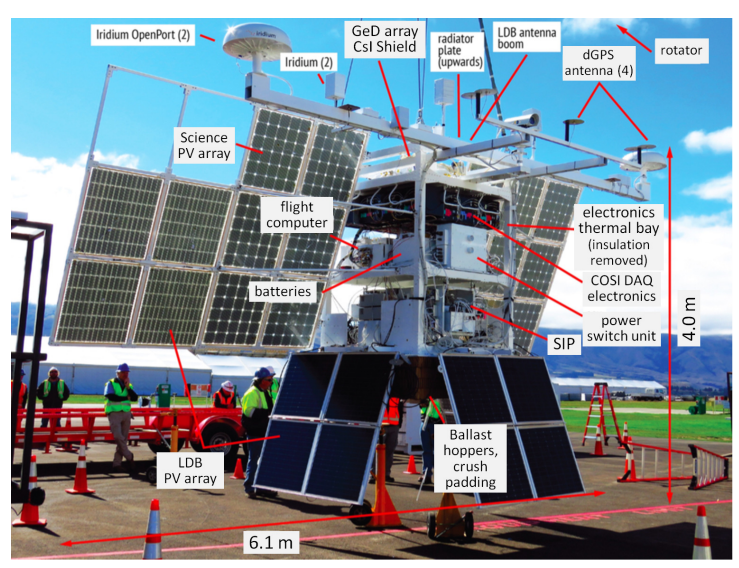

(b) COSI gondola

Figure 2: The configuration of COSI instrument: (a) the GeD array integrated in the cryostat with three of the four side CsI shield pieces; two more CsI shield pieces would be mounted in the bottom of the cryostat to reduce atmospheric background at float; (b) The 2016 COSI gondola fully integrated with the instrument. The side panels are removed to provide a view of the electronics bay, which is under the top tier where COSI cryostat is located.

to the rest of the electromagnetic spectrum; with improved sensitivity, COSI can answer open science question in this "MeV gap". With it's compact design and high spectral resolution, COSI is an excellent instrument to carry out polarization and nucleosynthesis studies. Flying in the southern hemisphere enables good exposure to the Galactic Center and Galactic plane. A brief summary about the three primary scientific goals for the COSI 2016 flight are below. Further details can be found in [7, 14].

Galactic Positron Annihilation The origin of the positron annihilation line from the Galactic Center region at $511 \mathrm{keV}$ has been a mystery since 1973 [8]. The key to understanding the anomalous, bright bulge emission lies in imaging and spectroscopy of the Galactic disk. To compare to the 511-keV map from INTEGRAL/SPI [9], COSI will produce the first direct image of the 511 $\mathrm{keV}$ line from the Galactic bulge and disk. Comparisons of the $511-\mathrm{keV}$ emission to ${ }^{26} \mathrm{Al}$ emission, as well as to emissions from other Galactic tracers observed by COSI, could lead to further understanding of the source of these positrons. In addition, the 511-keV spectral line profiles from the disk and bulge can be measured to study the annihilation mechanism.

Polarization of GRBs and Compact Objects Polarization measurements provide a unique diagnostic tool to determine emission mechanisms and source geometries. Different theoretical models predict different levels of polarization [10,11], and thus polarimetry can shed light on magnetic field ordering and the orientation of an accretion disk or jet. COSI takes advantage of the polarization dependence of Compton-scattering in high sensitivity to gain insight into these extreme environments. GRBs and bright gamma-ray compact objects, such as Galactic black holes and AGN, are the targets of interest for polarization studies. 


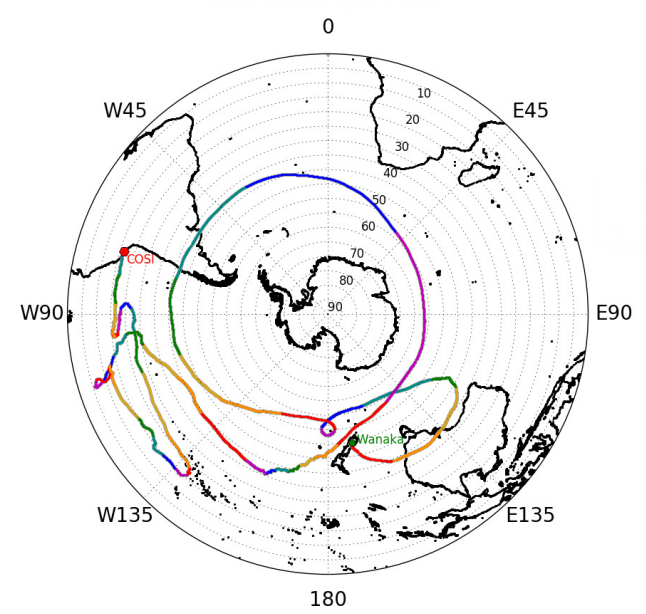

(a) Flight path

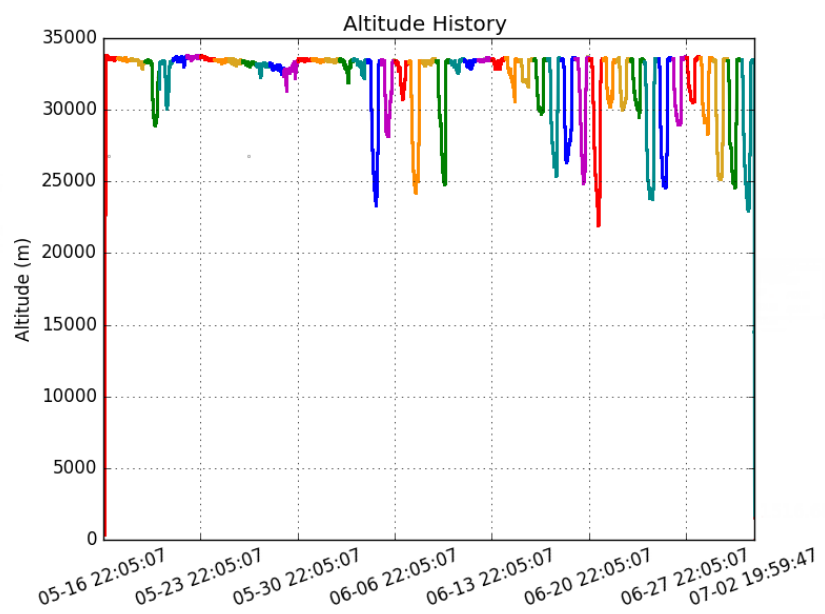

(b) Flight altitude history

Figure 3: The COSI 2016 flight: (a) path of COSI's 46-day flight, launching from Wanaka, New Zealand, and landing in Peru; (b) altitude history throughout the flight, where large altitude drops during the cold nights were seen after June 4th. The different rainbow colors trace the flight trajectory for each day.

Stellar Nucleosynthesis Several nuclear lines are only accessible in the soft gamma-ray band and are good tracers of stellar processes. ${ }^{26} \mathrm{Al}$ and ${ }^{60} \mathrm{Fe}$ are released into the interstellar medium during $\mathrm{ccSNe}$ and have $\sim$ Myr half-lives. With its $\sim 60 \mathrm{yr}$ half-life, ${ }^{44} \mathrm{Ti}$ is seen from young SNe. Mapping these diffuse emission lines and performing detailed spectroscopy reveal the dynamics of element formation, providing an understanding of SN nucleosynthesis. COSI's wide field imaging and high spectral resolution make it a great instrument for diffuse nuclear line measurements.

\section{Instrument and the 2016 Balloon Flight}

Instrument COSI consists of twelve germanium detectors (GeDs) that measure both the position and energy of gamma-ray interactions. The excellent spectral resolution and the moderate atomic number makes germanium a good detector material for Compton telescopes. Each GeD is a 37 $\times 37$ cross-strip planar detector, measuring $8 \times 8 \times 1.5 \mathrm{~cm}^{3}$, with 3 -D position sensitivity. The detectors were fabricated at Lawrence Berkeley National Laboratory using amorphous germanium contact technology [12], with a strip pitch of $2 \mathrm{~mm}$ and a $0.25 \mathrm{~mm}$ gap between strips. A 2$\mathrm{mm}$ thick guard ring surround an active area $\left(5400-\mathrm{mm}^{2}\right)$ on both faces of the detector to provide anticoincidence signals for rejection of events in these regions. The twelve detectors are stacked in a $2 \times 2 \times 3$ configuration (Fig. 1(b)) optimized for polarimetry and oriented towards the zenith. Further detail of the COSI GeDs are described in [13]. The GeD array, which is housed in an aluminum cryostat, is actively cooled with a Sunpower CryoTel CT free-piston Stirling cryocooler to hold the detectors operating at $83 \mathrm{~K}$.

CsI scintillators (4-cm thick each) surround the sides and bottom (two on the bottom and one on each side) of the GeD array to serve as an anticoincidence shield system (Fig. 2(a)), where events above $80 \mathrm{keV}$ are triggered to veto charged particles as well as photons incident on the in- 


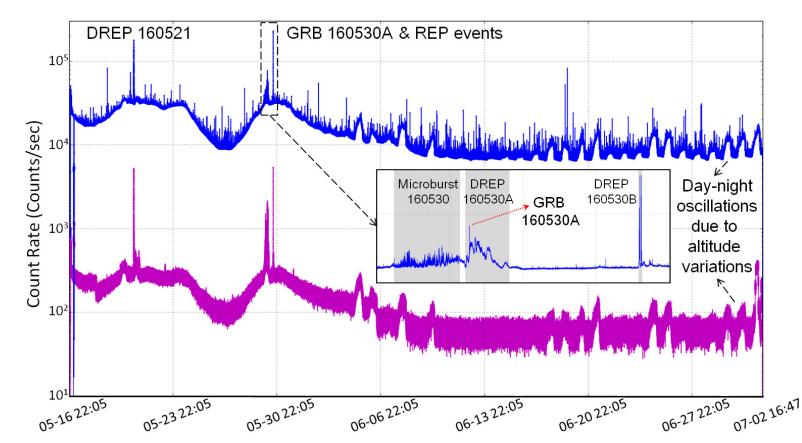

(a) Detector and shield rates

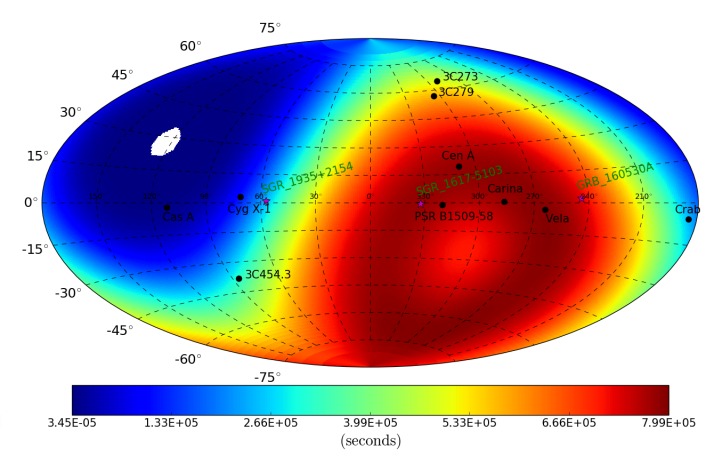

(b) COSI exposure

Figure 4: The COSI 2016 observation. (a) Trigger rates from the CsI shields and the sum of top GeDs rates throughout the flight. This shows the background variation due to different geomagnetic cutoffs (higher close to the magnetic South pole) and due to gamma-ray events. The medium-scale variations after June 4th are from altitude drops at night. GRB 160530A and multiple intense REP events were highlighted and annotated in the plot. (b) Total sky exposure during the COSI 2016 flight based on a simulated effective area at $356 \mathrm{keV}$.

strument from outside the FoV. The instrumental background, mainly atmospheric albedo photons, is therefore significantly reduced via the active shield even before the Compton imaging and other background-reduction techniques are applied.

A simple solar-oriented balloon gondola with reduced weight ( $\sim 240 \mathrm{~kg})$ was designed for COSI which can be easily repaired or replaced between flights. As the main mechanical support structure, the COSI gondola consists of a simple aluminum frame $\left(1.5 \times 1.5 \times 2.2 \mathrm{~m}^{3}\right)$ with three deck levels for instrument mounting, including an enclosed electronics bay for passive thermal control. The instrument mounts on the upper deck, with the instrument axis fixed in the zenith direction (Fig. 2(b)).

The 2016 Balloon Flight COSI was launched from Wanaka, New Zealand, on May 17th, 2016, and terminated above land $200 \mathrm{~km}$ north-west of Arequipa, Peru, on July 2nd. The flight path and altitude history of this successful 46-day flight are shown in Fig. 3. The unexpected drift to the North $\left(\sim 10^{\circ} \mathrm{S}, \sim 110^{\circ} \mathrm{W}\right)$ allowed for exposure of the Crab and Cyg $\mathrm{X}-1$ as well as measurements with lower atmospheric background. Altitude drops during cold nights is clearly seen after June 4th, resulting in early termination to guarantee recovery of the payload. The COSI instrument was successfully recovered on July 14th with no major damage. During the whole flight, nine of the GeD detectors worked flawlessly, while three failed at different times possibly due to highvoltage anomalies. The functionality of the high-voltage supplies and data acquisition electronics is currently being tested post-flight in Berkeley. More details about the 2016 flight and the COSI instrument are described in [14] and [3].

\section{Data Analysis Pipeline}

The data analysis pipeline of COSI was developed based on the Medium Energy Gamma-ray 


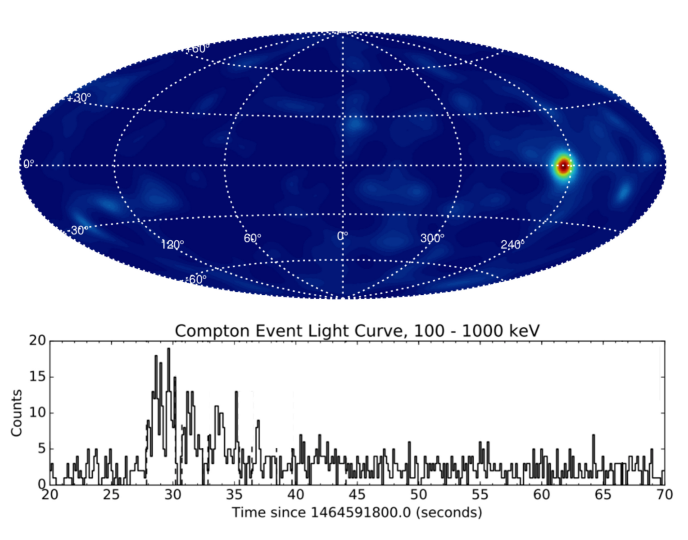

(a) Image and light curve

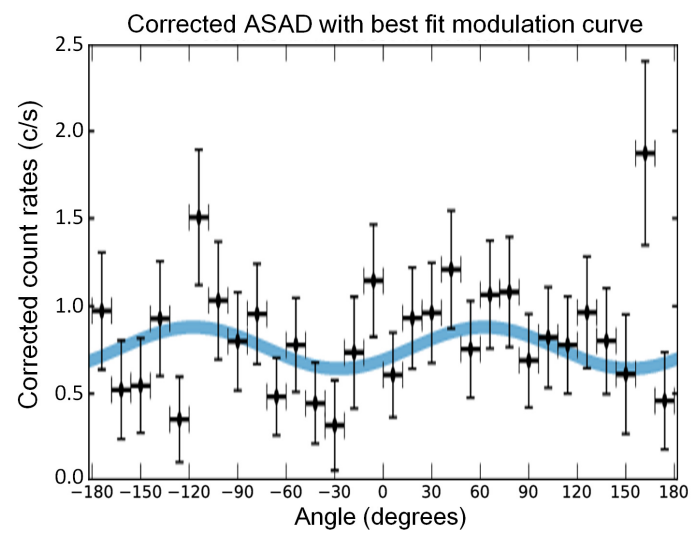

(b) Polarization modulation (ASAD)

Figure 5: COSI observation of GRB 160530A: (a) image showing the GRB localization in Galactic coordinates (top) and the light curve (bottom) of the burst; (b) the corrected azimuthal scattering angle distribution (ASAD) showing the polarization modulation of GRB 160530A.

Astronomy library (MEGAlib) toolkit [15], a set of software analysis tools providing programs for simulation, geometry modeling, event reconstruction, and image reconstruction. A detailed mass model was constructed for COSI to describe the instrument geometry, and a detector effects engine, which applies the intrinsic detector performance to Monte Carlo simulations, was developed to carry out performance benchmarking tests [16]. In-depth discussion of the energy and depth calibrations for COSI was presented in [19] and [20], and an overview of the polarization calibration was demonstrated in [21]. A response matrix, background model, and atmospheric absorption model are being assembled in XSPEC for further spectral analysis [26].

\section{Current Progress of Scientific Results}

The successful 46-day flight between $6^{\circ} \mathrm{S}$ and $56^{\circ} \mathrm{S}$ (Fig. 3(a)) provided COSI with a favorable exposure of the Galactic plane, the Galactic Center, and some of the brightest gamma-ray sources in the sky. Figure. 4(b) shows the exposure map from the entire flight based on a simulated effective area at $356 \mathrm{keV}$. In addition to astrophysical observations, the COSI 2016 flight may provide the most thorough study of mid-latitude gamma-ray backgrounds at balloon altitudes to date as well as unprecedented measurements of relativistic electron precipitation (REP) events.

GRB 160530A The bright GRB 160530A was detected by COSI in both the CsI and the GeDs [22]. Figure 5(a) shows an all-sky image localizing this long-duration ( $\sim 37 \mathrm{sec})$ GRB and its multipeak light curve. Wind/Konus and INTEGRAL/ACS [23] also detected GRB 160530A and verified COSI's absolute timing accuracy via the Inter-Planetary Network (IPN) [24]. The polarization characteristics of GRB 160530A was analyzed [24], and the resulting azimuthal scattering angle distribution (ASAD) can be found in Fig. 5(b). A maximum likelihood based technique was developed to improve our polarization sensitivity [25], resulting in a $90 \%$ confidence upper limit on the polarization level for GRB 160530A of 50-60\% [24]. COSI's observed spectrum was fit with 


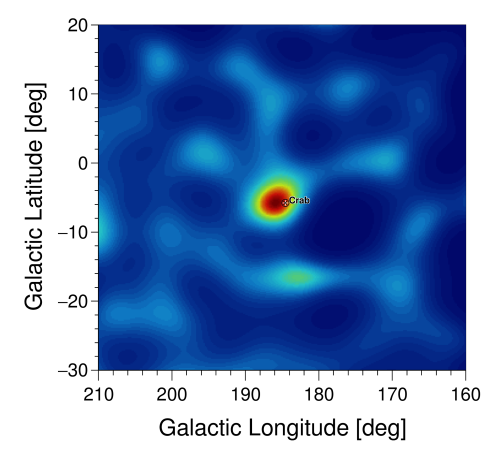

(a) Crab Pulsar \& Nebula

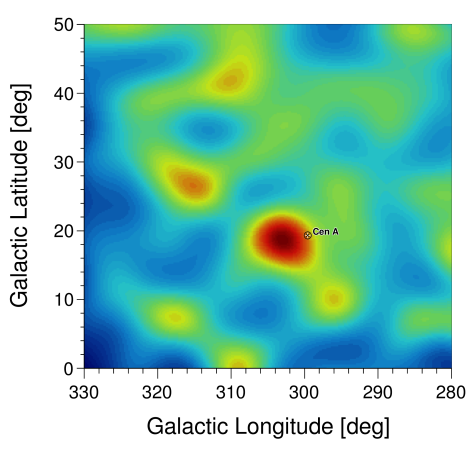

(b) Centaurus A

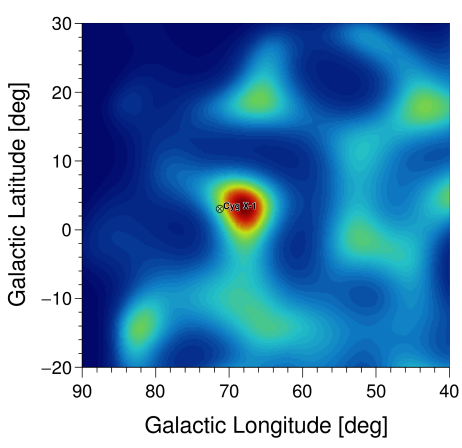

(c) Cygnus X-1

Figure 6: COSI observations of compact objects: (a) Crab pulsar and nebula; (b) Centaurus A (the closest AGN); (c) Cygnus X-1 (a galactic black hole).

a Band function model and corrected for atmospheric absorption. The result is in good agreement with the result from Wind/Konus [26].

Compact Object Detection The detection of bright, compact gamma-ray sources demonstrates the imaging capabilities of COSI. Preliminary images of the Crab Nebula, Cen A, and Cyg X-1 are shown in Figure 6. We started detailed spectral analysis with the brightest source, the Crab Nebula. Through modeling the detector response, background, and atmospheric absorption, in addition to event selections to optimize the source-to-background ratio, initial results of the Crab's spectral signature are consistent with measurements from other gamma-ray instruments [26]. The spectral analysis pipeline developed for the Crab will be applied to analyze Cen A (with longer exposure) and Cyg X-1 (with much lower exposure). Cen A is known for its giant lobes observed in both radio [17] and gamma-ray ( $>200 \mathrm{MeV})$ bands [18]. COSI will search for the extended feature from Cen A in the $\mathrm{MeV}$ band. Polarization studies on these compact objects are also ongoing based on the process implemented for GRB 160530A.

Galactic Positron Annihilation The 511-keV emission around Galactic Center has been detected significantly in the image and spectrum with COSI, marking the first detection of positron annihilation by a telescope with high spectral resolution and direct imaging capability. Figure 7 shows the preliminary background-subtracted spectrum which confirms a detection of the positron annihilation signal from a 16degree region around the Galactic center. The extension of the Galactic emission is being studied, and our flight results will be matched with accurate simulations of various Galactic positron annihilation distribution models. An image was obtained by including photon

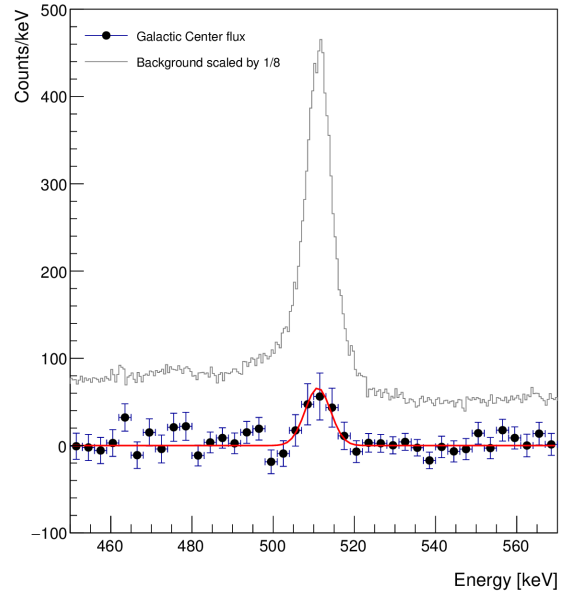

Figure 7: The preliminary backgroundsubtracted spectrum of the Galactic Center region around $511 \mathrm{keV}$. The raw spectrum is scaled down for reference. 
events around $511 \mathrm{keV}$ throughout the 46-day flight and shows a strong emission near the Galactic Center [27]. Imaging analysis is ongoing.

REP Events Although not a formal goal of the COSI mission, several REP events [28], which are due to bremsstrahlung gamma rays emitted from relativistic auroral electrons, were detected. Three duskside relativistic electron precipitation (DREP) events and a microburst precipitation event (Fig. 4(a)) were observed in this flight, and these are the first detections by an all-sky imaging gammaray detector. COSI's extraordinary performance in spectroscopy, timing, imaging, and polarimetry will help better understand these events.

CsI Shields Events The COSI CsI shield rates were used to trigger on possible GRB candidates in real-time. The automatic pipeline yielded corresponding all-sky images and light curves using Compton photon events within a $\sim 500$-sec window centered around the alert triggered time, and each alert was then verified manually. There were several confirmed GRBs and flaring activities from Soft Gamma-ray Repeaters (e.g. SGR 1935+2154, SGR 1617-5103 were active during this flight) (Fig. 8). The raw data is being scanned for SGR flares and possible GRB events missed with the in-flight trigger mechanism.

\section{Future Work}

The COSI instrument is currently being repaired and tested in anticipation of a future launch opportunity, while the collaboration is working on
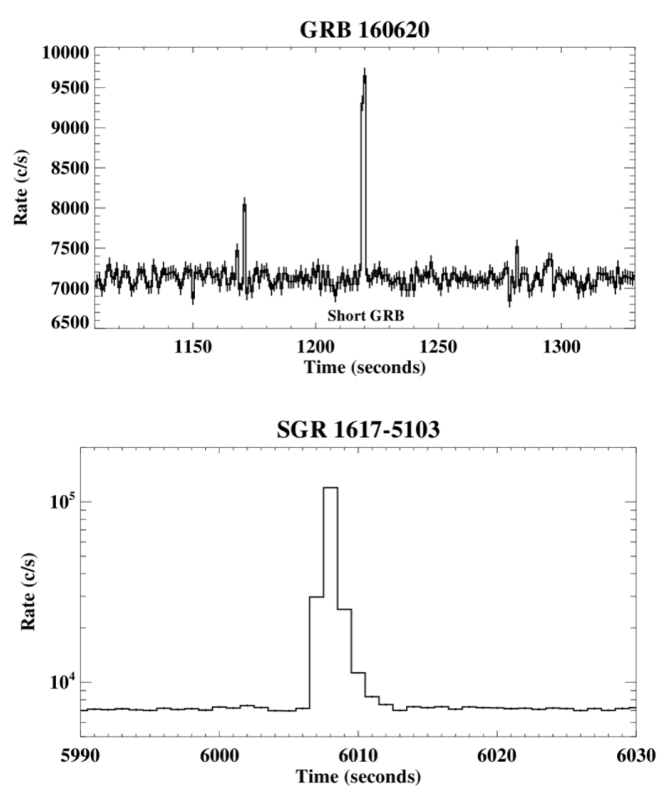

Figure 8: CsI shield events (GRB and SGR). Minor peaks in top figure are cosmic rays or delayed fluorescence in the CsI shields future upgrades. The most desirable upgrade will be pushing the GeD strip to a finer pitch of $0.58 \mathrm{~mm}$, which is anticipated to achieve an angular resolution of 1.5 degree at $1.809 \mathrm{MeV}$. In order to meet the readout requirements of the finer-strip COSI detectors, a Germanium Front-End ASIC is being developed in a NASA ROSES program led by NRL. In addition, Dr. Andreas Zoglauer in the COSI collaboration will be working on a NASA-funded project to enhance our Compton image reconstruction capabilities.

\section{Acknowledgments}

Support for COSI is provided by NASA grant NNX14AC81G and Taiwan MOST grant 1052112-M-007-002.

\section{References}

[1] Boggs, S. E., et al., New Astronomy Reviews 48, 251 (2004). 
[2] Schonfelder, V., Aarts, H., Bennett, K., Astrophys. J. Supplement 86, 657 (1993).

[3] Chiu, J.-L., et al., Nucl. Instrum. Meth. A, 784, 359-363 (2015).

[4] Bowen, J. D., et al., AAS HEAD Meeting, 38, 385 (2006).

[5] Bandstra, M. S., et al., IEEE Nucl. Sci. Symp. Conf. Rec., 2131-2139 (2009).

[6] Bandstra, M. S., et al., Astrophys. J., 738(1), 9 (2011).

[7] Boggs, S. E., et al., arXiv:astro-ph/0608532 (2006)

[8] Johnson, W. N., III and Haymes, R. C., Astrophys. J. 184, 103-125 (1973).

[9] Bouchet, L., Roques, J. P. and Jourdain, E., Astrophys. J. 720, 1772-1780 (2010).

[10] Lei, F., Dean, A. J. and Hills, G. L., Space Sci. Rev., 82(3-4), 309-388 (1997).

[11] Toma, K., et al., Astrophys. J. 698, 1042-1053 (2009).

[12] Amman, M., Luke, P. N., and Boggs, S. E., Nucl. Instrum. Meth. A., 579(2), 886-890 (2007).

[13] Coburn, W., et al., Proc. SPIE 4784, 54 (2003).

[14] Kierans, C. A. et al., in proceedings of 11th INTEGRAL Conference, PoS (INTEGRAL2 016 ) 075 (2016)

[15] Zoglauer, A. et al., New Astronomy Reviews 50, 629 (2006).

[16] Sleator, C., et al., in proceedings of 11th INTEGRAL Conference, POS ( INTEGRAL2016) 087 (2016)

[17] Combi, J. A., \& Romero, G. E., A\&AS, 121, 11 (1997)

[18] Abdo, A. A., et al., Science, 328, 725 (2010)

[19] Kierans, C. A. et al., Proc. SPIE 9144, 91443M (2014).

[20] Lowell, A., et al., Proc. SPIE 9915, 99152H (2016).

[21] Bellm, E. C., et al., IEEE Nucl. Sci. Symp. Conf. Rec., 444-448 (2009).

[22] Tomsick, J. A., et al., GCN Circular, vol. 19473 (2016).

[23] Svinkin, D., et al. GCN Circular, vol. 19476 (2016).

[24] Lowell, A., et al., paper 1 (submitted to ApJ).

[25] Lowell, A., et al., paper 2 (submitted to ApJ).

[26] Sleator, C., et al., (in prep).

[27] Kierans, C. A. et al., (in prep).

[28] Millan, R. M. and Thorne, R. M., J. Atmos. Sol.-Terr. Phys. 69(3), 362-377 (2007). 